\title{
Studies of Glycine Guanidine Nitrate single crystals In the Aqueous Solution of $\alpha$-Glycine and Guanidine Nitrate.
}

\author{
D. Arul Asir Abraham*, U. Sankar ${ }^{\mathrm{b}}$, S. Perumal ${ }^{\mathrm{c}}$, P.Selvarajan ${ }^{\mathrm{d}}$ \\ ${ }^{a}$ Department of Physics, St. John's College, Palayamkottai-627002, Tamil Nadu, India. \\ ${ }^{b}$ Department of Physics, Sri K.G.S Arts College, Srivaikuntam-628601, Tamil Nadu, India. \\ ${ }^{c}$ Physics Research Centre, S.T. Hindu College, Nagercoil-629003, Tamil Nadu, India. \\ ${ }^{d}$ Department of Physics, Aditanar College of Arts and Science, Tiruchendur- 628216, Tamil nadu, India.
}

\begin{abstract}
The field of nonlinear optics became practically a reality after the invention of laser. High Performance electro-optic switching elements for telecommunications and optical information processing are based on materials with high nonlinear optical $(N L O)$ properties. The development and encroachment of high technology, from transportation, computation to information is based on the availability of materials in the form of single crystals. An semi organic nonlinear optical single crystal of Glycine Guanidine Nitrate $(G L G N)$ has been grown by the aqueous solution of $\alpha$-Glycine and Guanidine Nitrate by slow evaporation of the saturated solution at room temperature. Single crystal of (GLGN), an semi organic nonlinear optical (NLO) material, has been grown by slow solvent evaporation technique. Good optical quality single crystals with dimension up to $31 \times 30 \times 7 \mathrm{~mm}^{3}$ are obtained. Single crystal X-ray diffraction $(X R D)$ studies revealed the presence of monoclinic structure with space group. The crystals are characterized by optical absorption spectrum, FTIR and X-ray diffraction studies The mechanical and Differential thermal analysis (DTA) and thermo gravimetric analysis (TGA) study of the grown crystals are also investigated.
\end{abstract}

Key Words: single crystal, X-Ray diffraction, FTIR, thermo gravimetric/differential thermal analyzer.

\section{Introduction}

The nonlinear optical (NLO) materials play a major role in nonlinear optics and in particular they have a great impact on information technology and industrial applications. In the last decade, however, this effort has also brought its fruits in applied aspects of nonlinear optics.

This can be essentially traced to the improvement of the performances of the NLO materials. There has been a growing interest in crystal growth process, particularly, in view of the increasing demand for materials for technological applications [1-3]. The wide range of applicability of single crystals is evident in the fields of semiconductors, polarizer's, infrared detectors, solid state lasers, nonlinear optic, piezoelectric, acousto-optic, photosensitive materials and crystalline thin films for microelectronics and computer industries. The growth of single crystals and their characterization towards device fabrication have assumed great impetus due to their significance in both academic research and applied research. Among the organic materials, amino acids constitute a family in which glycine is the simplest of all the amino acids. It has been reported that some complexes of amino acids with some simple inorganic salts may exhibit ferroelectric properties [4-7]. Also, the semi organic compounds have greater efficiency than the KDP crystals [8]. Glycine added the aqueous solution of Guanidine nitrate is one of the complexes of glycine and it possesses the Non-linear Optical (NLO) properties. This material can find useful applications in the field of opto-electronics. This material is characterized in order to find the suitability of the required applications. Hence, in the present investigation, the growth aspects of the Glycine added in the aqueous solution of Guanidine Nitrate (GLGN) crystals have been carried out using solvent evaporation technique. The grown crystal is subjected to different characterizations such as single X-ray diffraction (XRD), Fourier transform infrared transmission (FTIR), Ultraviolet (UV), and thermo gravimetric Analyzers /Differential Thermal Analyzer (TGA/DTA) studies were carried out for the grown crystals.

Abbreviations: FTIR, Fourier transform infrared transmission; XRD, X-ray diffraction; GLGN, Glycine Guanidine Nitrate; TG/DTA, Thermo gravimetric/Differential Thermal Analyzer.

\subsection{Synthesis}

\section{Experimental Procedure}

Colorless single crystals of GLGN were grown, from aqueous solution by slow evaporation technique. The starting materials was analytical grade reagents of $\alpha$-glycine added with the aqueous solution of Guanidine nitrate. The aqueous solution was prepared by dissolving equimolar amounts of glycine and Guanidine nitrate in deionized water by slow evaporation at ambient temperature. The solution was allowed to evaporate slowly. Small transparent colorless single crystals were obtained through spontaneous 
nucleation. By selecting macro defect free crystals as seeds, single crystals were grown to a dimension of $31 \times$ $30 \times 7 \mathrm{~mm}^{3}$ at ambient temperature as shown in Figure 1, In this technique, during the growth of GLGN, several funguses like organism were formed in the solution. These organisms initially started on the surface and gradually after a few days, sinked into the solution, thereby contaminating it, and the growth of the crystals to larger dimension is prohibited. The growth of these microbes is prevented by adding a few drops of hydrogen peroxide $\left(\mathrm{H}_{2} \mathrm{O}_{2}\right)$.

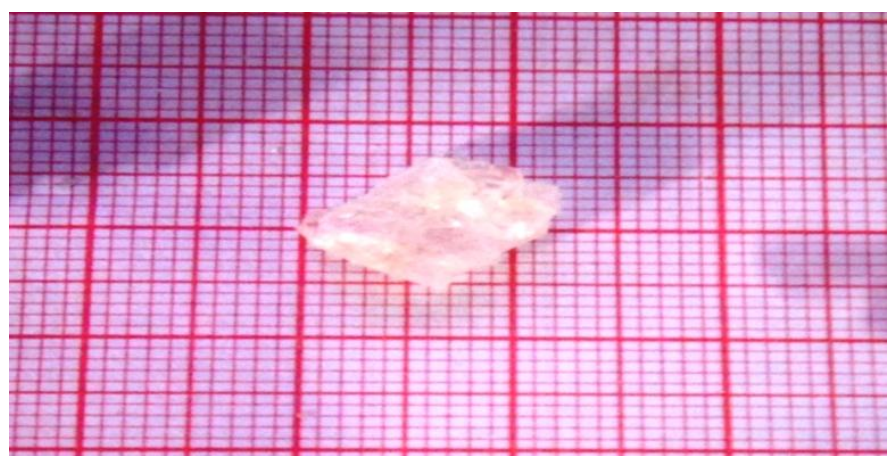

Fig 1. Grown single crystal of GLGN

\subsection{Solubility determination}

The solubility of GLGN was determined by dissolving the synthesized product in Millipore water in an airtight container kept in a constant temperature bath, the content was continuously stirred for 2-3 hours. After attaining the saturation, the equilibrium concentration of the solute was estimated gravimetrically. The same process was repeated for different temperatures $\left(30,35,40,45,50\right.$ and $\left.55^{\circ} \mathrm{C}\right)$. The variation of solubility with temperature Fig. 2 indicates that glycine with Guanidine Nitrate have high positive solubility-temperature co-efficient values.

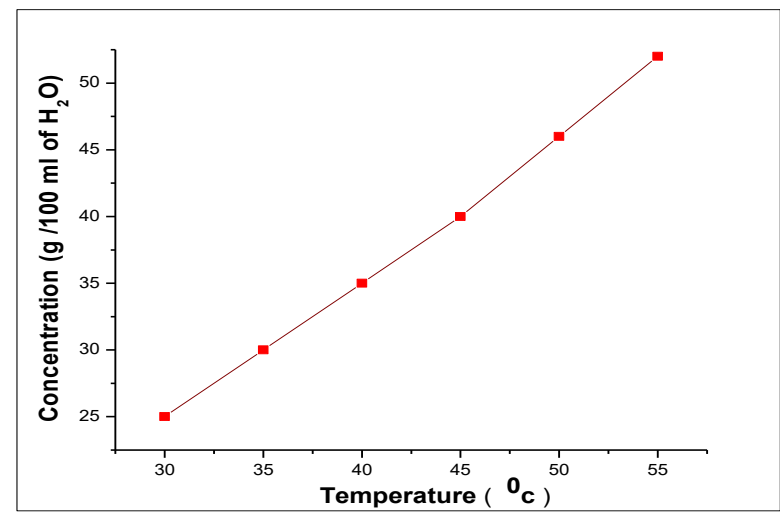

Fig 2. Solubility curve of GLGN Crystal

\subsection{Single crystal X-ray diffraction (XRD)}

\section{Results And Discussion}

The single crystal XRD analysis for the grown crystals has been carried out to identify the lattice parameters. The calculated unit cell lattice parameters are $\mathrm{a}=5.095(4) \AA, \mathrm{b}=11.926(15) \AA, \mathrm{c}=5.451(2) \AA$, volume $=308.0(4) \AA^{3}$, and the Wavelength is $0.71073 \AA$. The crystal belongs to monoclinic structure with space group Cc with $\alpha=90^{\circ}, \beta=111.56(5)^{\circ}$ and $\gamma=90^{\circ}$. This is in close agreement result by Karolin et al [9].

\subsection{Powder XRD analysis}

The Powder XRD pattern of the grown crystals was recorded using D8 Advanced Bruker Powder Xray Diffractometer. The positions of the peaks are found to be matching with the literature. Fig. 3 depicts the powder XRD pattern of the glycine with Guanidine Nitrate crystals and are found to be in good agreement with literature [10]. 


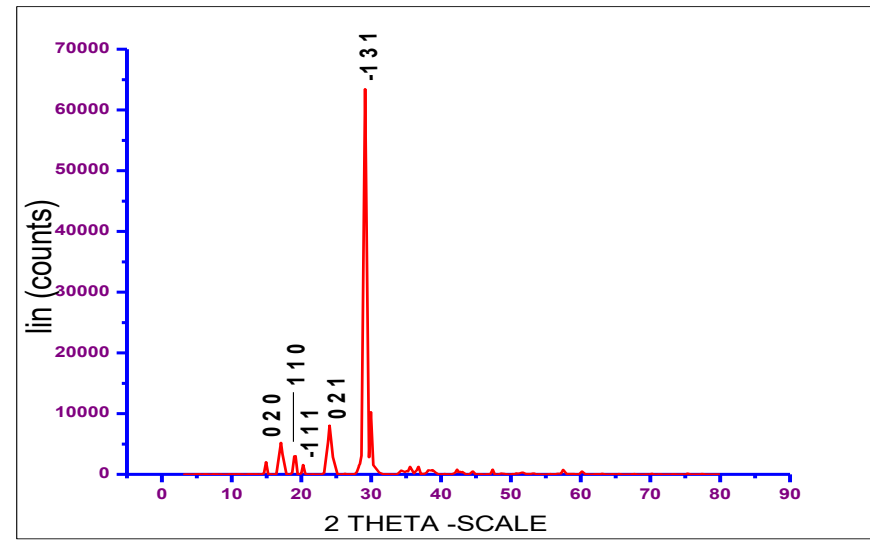

Fig 3. Powder XRD of GLGN crystals

\subsection{Fourier transforms infrared transmission (FTIR) spectral analysis}

The FTIR analysis of GLGN crystal was carried out between $400-4000 \mathrm{~cm}^{-1}$ using Perkin Elmer RX 1 spectrometer and the resulting spectrum is shown in Figure 4. The spectrum shows that the glycine molecule exists as a dipolar ion in which the carboxyl group is present as a carboxylate ion and amino group exists as ammonium ions. The peak at $3409.69 \mathrm{~cm}^{-1}$ and 3202.09 is due to $\mathrm{NH}_{3}{ }^{+}$asymmetric stretching vibrations. The peak at $2821.25 \mathrm{~cm}^{-1}$ is due to N-C stretching vibration. The peak at $2407.86 \mathrm{~cm}^{-1}$ is assigned to $\mathrm{C}-\mathrm{H}_{2}$ asymmetric stretching. The peak at $2215 \mathrm{~cm}^{-1}$ is due to $\mathrm{NH}_{3}{ }^{+}$asymmetric stretching and torsional vibrations. The peak at $1777.07 \mathrm{~cm}^{-1}$ is due to $\mathrm{NH}_{3}{ }^{+}$asymmetric bending. The peak at $1377.32 \mathrm{~cm}^{-1}$ is due to COO symmetric stretching vibration. The peak at $1160.05 \mathrm{~cm}^{-1}$ is due to carboxylate group of GLGN a symmetric stretching vibration [11]. The peak at $824.99 \mathrm{~cm}^{-1}$ is assigned to $\mathrm{C}-\mathrm{CH}_{2}$ bending vibration. The peak at $534.53 \mathrm{~cm}^{-1}$ is assigned to $\mathrm{NH}^{+}$Torsion asymmetric stretching. The FTIR assignments and the modes of vibrations are listed in Error! Reference source not found.1.

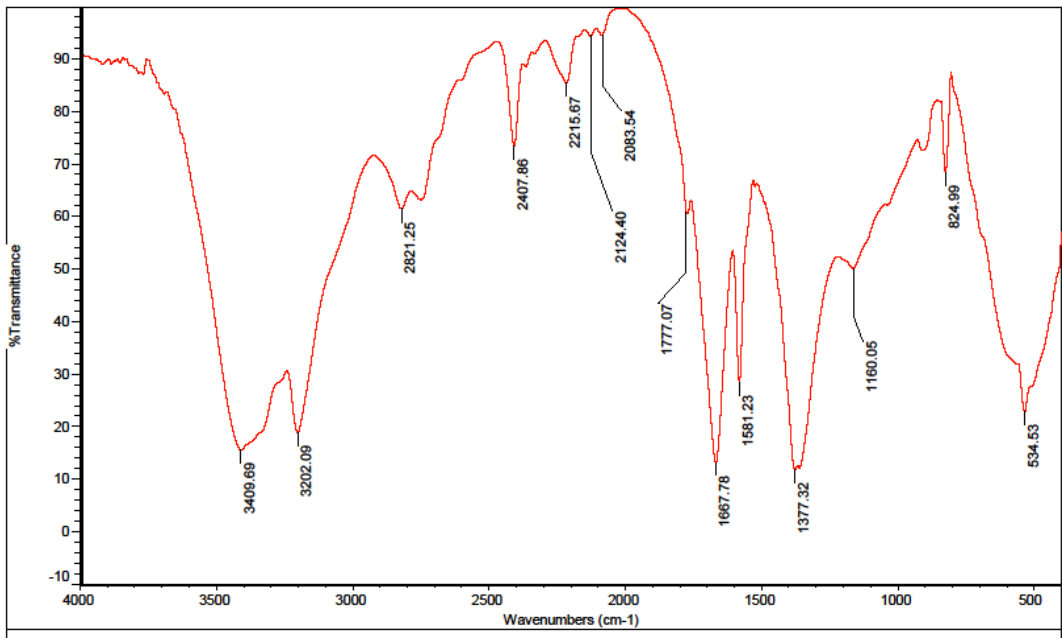

Fig. 4 FT-IR Spectrum for GLGN

Table 1. FTIR assignments of GLGN

\begin{tabular}{|l|l|l|}
\hline S.No. & Wave no. $(\mathrm{cm}-1)$ & Assignment \\
\hline 1 & 3409,3202 & $\mathrm{NH}_{3}{ }^{+}$asym str \\
\hline 2 & 2821.25 & $\mathrm{~N}-\mathrm{C}$ str \\
\hline 3 & 2407.86 & $\mathrm{C}-\mathrm{H}_{2}$ asym str \\
\hline 4 & 2215 & $\mathrm{NH}_{3}{ }^{+}$asym str and torsion \\
5 & 2124,2063 & Combination bond \\
\hline 6 & 1777 & $\mathrm{NH}_{3}{ }^{+}$asym bend \\
7 & 1667 & $\mathrm{NH}_{3}{ }^{+}$bending \\
\hline 8 & 1377.32 & $\mathrm{COO}$ sym str \\
\hline 9 & 1160.05 & $\mathrm{CCN}$ a sym str \\
\hline 10 & 824 & $\mathrm{C}-\mathrm{CH}_{2}$ bend \\
\hline 11 & 534.53 & $\mathrm{NH}^{+}$Torsion \\
\hline
\end{tabular}




\subsection{UV-Vis Spectroscopy}

\subsubsection{Absorption Spectroscopy}

The optical absorption spectrum of GLGN crystal of thickness $1 \mathrm{~mm}$ was measured in the range of 190-1100 nm and the spectrum is shown in Error! Reference source not found.5. It is observed that the absorbance is low throughout the visible and IR region. The cut-off wavelength of the GLGN crystal is found to be $203 \mathrm{~nm}$.

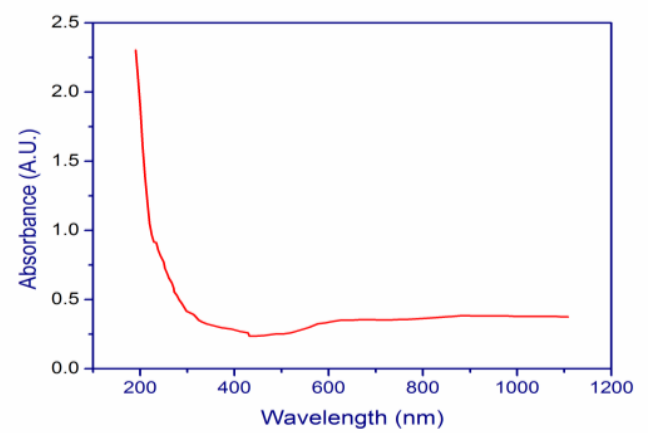

Fig 5. UV-Vis absorption spectrum of GLGN

\subsubsection{Transmission Spectroscopy}

The UV-Vis transmittance study on GLGN was carried out using PerkinElmer's LAMBDA 35 UV-Vis Spectrophotometer for the wavelength range of 190-1100 nm. The observed transmittance spectrum of GLGN is shown in Error! Reference source not found.6. The crystal shows very good transmittance in the entire visible range and is sufficiently good for SHG [12].

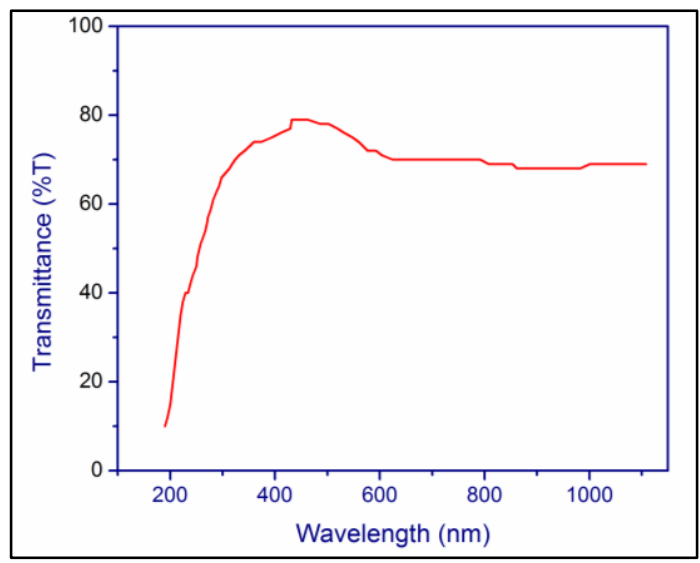

Fig 6.UV-Vis transmission spectrum of GLGN

\subsection{Second Harmonic Generation by Kurtz Powder Technique}

Second harmonic test on GLGN sample was done by Kurtz and Perry powder technique [13]. This technique has been considered as an authentic experimental method to verify the symmetry of a crystalline material. The microcrystalline powdered sample was packed in a capillary tube of diameter $0.154 \mathrm{~mm}$. The powder sample with an average particle size of 100-150 $\mu \mathrm{m}$ was illuminated using a Q-switched mode-locked Nd:YAG laser of pulse width $10 \mathrm{~ns}$ at a wavelength of $1064 \mathrm{~nm}$ and $10 \mathrm{~Hz}$ fundamental radiation. When a laser input energy $.8 \mathrm{j}$ was passed through the sample and KDP, the output signal obtained from GLGN was $3.6 \mathrm{mj}$ and KDP was 2. 2mj.Thus it concluded that the second harmonic generation efficiency of GLGN is 1.6 times of KDP.

\subsection{Thermal analysis}

The Thermogravimetric analysis on GLGN crystal was performed with the help of TA instruments, Q600 SDT for a temperature from $40^{\circ} \mathrm{C}$ to $640{ }^{\circ} \mathrm{C}$ at $10{ }^{\circ} \mathrm{C} / \mathrm{min}$. The DSC, TGA thermogram of GLGN is shown in Error! Reference source not found.7.

The sample remained stable up to $225^{\circ} \mathrm{C}$ [14]. However, there is a weight loss of about $7.42 \%$ between $212^{\circ} \mathrm{C}$ and $248.03{ }^{\circ} \mathrm{C}$. In the Glycine Guanidine Nitrate (GLGN) compound, the contribution of water 
is $7.65 \%$. Therefore, the weight loss in the first stage $(7.42 \%)$ is due to the liberation and evaporation of water from the compound. The DTA curve has an endothermic peak at $298.84{ }^{\circ} \mathrm{C}$. This may be considered as the decomposition point of the compound. But immediately after this, i.e. at $299{ }^{\circ} \mathrm{C}$, an abrupt weight loss of $88.28 \%$ is observed. From this it is understood that almost the entire residual GLGN compound gets evaporated. This happens before the temperature reaches $301{ }^{\circ} \mathrm{C}$ and a trivial residue of nearly $4.3 \%$, remains till the end of the observation $\left(6400{ }^{\circ} \mathrm{C}\right)$. Thus, it is concluded that GLGN sublimates at $299.1{ }^{\circ} \mathrm{C}$ is shown in fig 8.

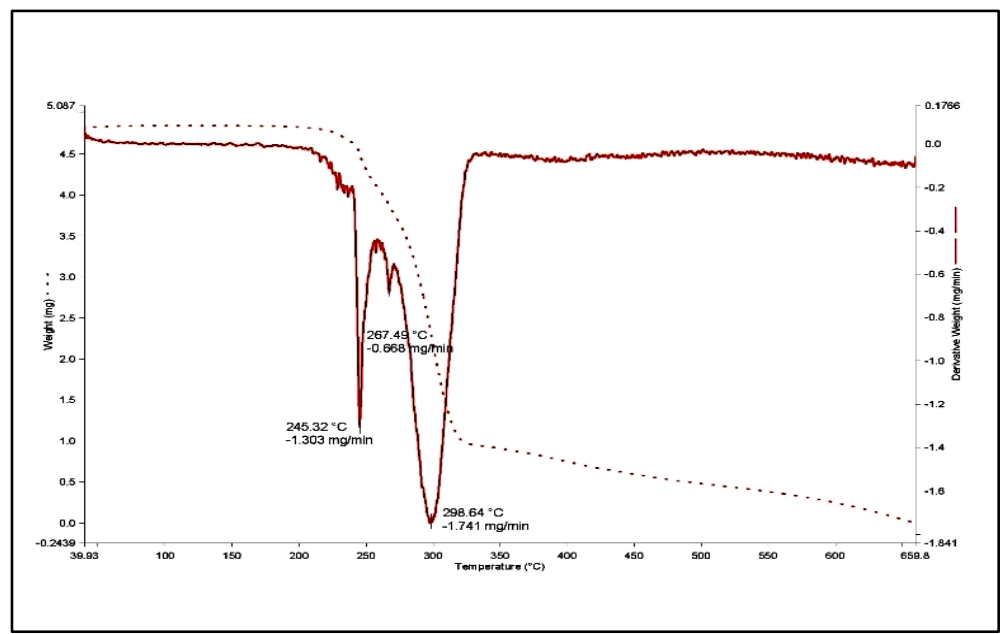

Fig 7.TGA analysis curve of GLGN

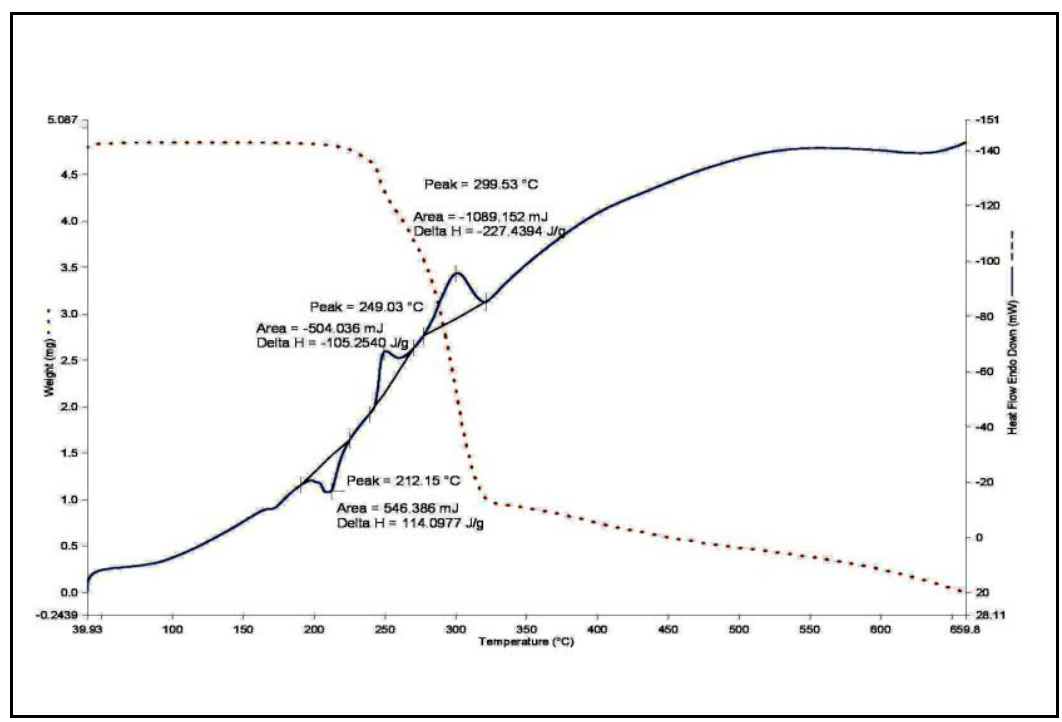

Fig 8.DTA analysis curve of GLGN

\subsection{Micro hardness Analysis}

The Vickers micro hardness of the grown GLGN crystal was determined using SHIMADZU HMV-2T micro hardness tester. The indentations were made on the polished crystal for the loads of 25,50 and $100 \mathrm{~g}$. The indentation time was kept as $5 \mathrm{~s}$ for all the loads. The graph showing variation in HV with load is shown in Error! Reference source not found.9. it belongs to soft material category [15]. 


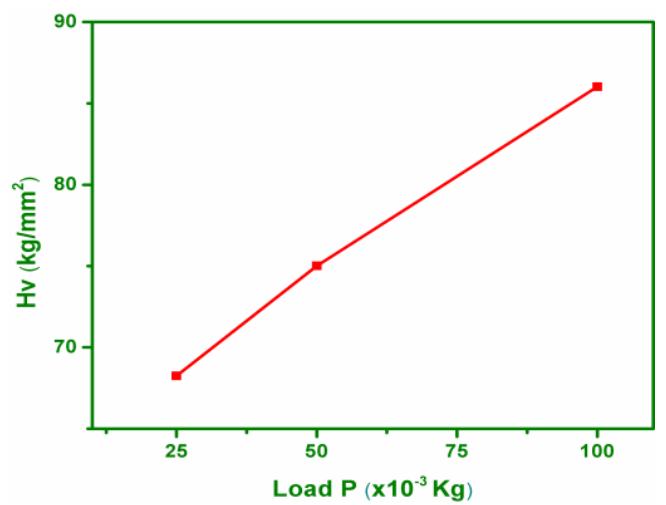

Fig9.Variation in the Vickers hardness HV with load P of GLGN

IV. Conclusion

Transparent single crystals of GLGN have been grown successfully using slow solvent evaporation technique. X-ray analysis reveals that GLGN crystallizes into the monoclinic system with space group Cc. UV-Visible absorption spectrum shows excellent transmission in the entire visible region. The FTIR spectrum shows that glycine molecule exists as a dipolar ion in which the carboxyl group is present as carboxylate ion and amino group is present as ammonium ion also their functional groups were identified. TG and DTA thermograms reveal that the sample is highly stable up to $225^{\circ} \mathrm{C}$.The SHG efficiency shows the GLGN has high NLO property.

\section{References}

[1] R.A.Laudise, R.Ueda and J.B.Millin, Crystal Growth and Characterization, North- Holland Publishing Co, (1975).

[2] J.C. Brice, 'Crystal Growth Processes', Halsted Press, John Wiley and sons, New York, (1986).

[3] H.S.Nalwa and S.Miyata S, 'Nonlinear Optics of Organic Molecules and Polymers', CRC Press Inc., New York, (1996).

[4] Deepthy A, Bhat HL (2001).Growth and Characterization of ferroelectric Glycine Phosphite Single Crystals, J. Cryst. Growth, 226-287

[5] Hoshino S, Mitsui T, Jona F, Pepinsky R (1957). Crystal Structure of the Ferroelectric Phase of (Glycine) 3·H2SO4 Phys. Rev., $107-125$

[6] Natarajan S, Mohan Rao JK (1984). Crystal Structure of bis (glycine) Calcium (II) dichloride tetrahydrate, Z. Kristallogr. $152-179$.

[7] Natarajan S, Ravikumar K, Rajan SS (1984). Crystal Structure of diaqua Nitrato Glycine Calcium (II) Nitrate, Z. Kristallogr., 168175 .

[8] J.Hernandez-paredez,D.Glossman-Mitnik,H.E.Esparsa-ponce,M.E.Alwarez-Ramosand A.Duarte-Muller “ Band structure,optical properties and infrared spectrum of glycine sodium nitrate crystal",journal of molecular structure, vol .875, no.1-3,pp 008

[9] A.Karolin.K.Jayakumari,C.K.Mahadevan,Growth and characterization of pure $\mathrm{NI}^{2+}$ doped glycine sodium sulphate crystals,int jour of Res and Tech vol:02,issue:12(2013) pp:646651

[10] Ra.Shanmugavadivu,G.Ravi,A.Nixon Azariah,j physics and chemistry of solids., 2006,67,1858-1861.

[11] T.Balakrishnan and K.Ramamurthi,Cryst.Res.Technol,41, no 12.1184-1186(2006).

[12] J.Ramamoorthy and S.Dhanuskodi.spectrochemica Acta part A.,2007,68,1213.

[13] S.K.Kurtz,T.T.Perry,j.Appl.phys.1968.,39,3798.

[14] M.Lenin,M.Chandrasekar,G.Udayakumar., Growth and characterization of glycine zinc sulphate,int.jour.of chem. Tech,vol.6.no.5,pp 2683-2688.

[15] E.M.Onitsch, Mikroskopie 2,1941,135 